\begin{tabular}{|c|c|c|c|c|c|c|}
\hline No & $\begin{array}{l}\text { Age } \\
\text { (Years) }\end{array}$ & $\begin{array}{l}\mathrm{HbA1c} \\
(\mathrm{mmol} / \mathrm{mol})\end{array}$ & $\begin{array}{l}\text { Average } \\
\text { number of } \\
\text { BG tests } \\
\text { per day } \\
\text { (Actually done) }\end{array}$ & $\begin{array}{l}\text { Average } \\
\text { number of } \\
\text { BG tests per } \\
\text { day (manual } \\
\text { entries) }\end{array}$ & $\begin{array}{l}\text { Mean BG } \\
(\mathrm{mmol} / \mathrm{L})\end{array}$ & $\begin{array}{l}\text { Mean BG } \\
\text { after removal } \\
\text { of manual } \\
\text { entries (mmol/L) }\end{array}$ \\
\hline 1 & 13.4 & 66 & 2.4 & 1.2 & 11.3 & 12.3 \\
\hline 2 & 14.9 & 72 & 0.4 & 4.5 & 7.2 & 12.3 \\
\hline 3 & 15.1 & 65 & 4.7 & 3.1 & 8.8 & 10.6 \\
\hline 4 & 15.2 & 56 & 0.5 & 5.3 & 8.6 & 13.4 \\
\hline 5 & 16.2 & 69 & 3.1 & 1.6 & 10 & 11.6 \\
\hline
\end{tabular}

Conclusion $84.6 \%$ of patients adhered to the expected number of BG tests while a $15.4 \%$ did not. The negative correlation between the number of BG tests and mean BG levels confirms the importance of frequent $B G$ monitoring to improve glycaemic control. 6.4\% (mainly adolescents) were manually entering fictitious BG levels which offered false assurance to family and clinician. Diabetic teams should be aware of this and vigilant to detect noncompliance in adolescents on insulin pump and offer appropriate counselling.

\section{G480(P) CARBOHYDRATE COUNTING FROM DIAGNOSIS: IS IT PRACTICAL, ACCEPTABLE AND EFFECTIVE?}

TP Candler, S Blakey, S Matthai. Paediatrics, Gloucestershire Royal Hospital, Gloucester, UK

\subsection{6/archdischild-2015-308599.434}

Background Carbohydrate counting has become a principal strategy for children with type1 diabetes. Precision with carbohydrate counting are associated with lower HbA1C in children with type 1 diabetes (Mehta, 2009). Traditionally, children were not taught this during their first presentation with diabetes. In Gloucester and our network, carbohydrate counting is being taught from diagnosis to embed this practice early.

Aims With regards early carbohydrate counting education, we asked: is it practical? is it acceptable to families? is it effective?

Methods We compared two 12 month time periods 1/3/12-28/ $2 / 13$ (education delayed) and 1/3/13-28/2/14 (education early). A sample of 15 patients from both years had their notes and biochemistry results examined. A questionnaire about patient's experience of early carbohydrate counting was sent.

Results Comparing the two time periods (delayed vs. early); the mean age at diagnosis was 9.07 vs. 9.33 years, $36 \%$ vs. $47 \%$ of patients presented in DKA. The majority started on basal bolus regime but $14 \%$ vs. $7 \%$ of patients were started on a pump during first admission. There was no significant difference in length of stay (4.21 vs. 5.13 days, $\mathrm{p}=0.056$ ). Falls in HbA1C appear sustained across the early education group. 81\% learnt $\mathrm{CHO}$ counting within a week, $64 \%$ preferred to learn $\mathrm{CHO}$ counting in hospital and $100 \%$ found the education useful.

Conclusion Early education about carbohydrate counting appears both practical and effective. There was no increase in length of stay between the two groups. There was generally positive feedback from those families that had early $\mathrm{CHO}$ counting education.

\section{G481(P) INSIGHTS INTO FACTORS AFFECTING CHILDHOOD OBESITY IN UNITED KINGDOM MIGRANTS OF SOUTH ASIAN ORIGIN}

${ }^{1} \mathrm{LMC}$ Burland, ${ }^{2} \mathrm{H}$ Hassanzadeh, ${ }^{3} \mathrm{BL}$ Green. ${ }^{1}$ Department of Paediatrics, Bradford Teaching Hospital NHS Trust, Bradford, UK; ${ }^{2}$ Epsom and St Helier University Hospitals NHS Trust, Carshalton, UK; ${ }^{3}$ Leeds Teaching Hospitals NHS Trust, Leeds, UK

\subsection{6/archdischild-2015-308599.435}

Introduction Childhood obesity represents a significant threat to the health and wellbeing of future generations of adults in the United Kingdom (UK). Certain ethnic minorities including those of South Asian origin experience notably higher rates of childhood obesity. As the primary care-providers in South Asian culture, mothers play a significant role in determining child health: improved understanding of perceptions regarding childhood obesity may therefore facilitate the development of targeted public health strategy. This study aimed to determine the views of South Asian mothers regarding childhood obesity.

Methods A purposive sampling strategy was used to identify mothers of children aged 3-11 years of South Asian origin attending a childrens' day centre in Bradford, UK. Eligible mothers were consented for participation in semi-structured in-depth interviews regarding childhood obesity. Interviews were facilitated by an experienced qualitative researcher in the presence of a dedicated translator. All conversations were electronically recorded and independently reviewed for retrospective quality control. Thematic and content analysis was applied in order to generate coding themes for analysis in order to facilitate indexing and summarisation of key data.

Results A total of 14 mothers, aged $32.4 \pm 6$ years were interviewed. Thematic and content analysis generated four distinct themes: 1) perception of childhood obesity; 2) implications for long-term health; 3) awareness of preventative measures; and 4) barriers to prevention. Participants reported reasonable awareness of obesity as a risk factor for future health problems but knowledge of specific disease states was limited. Understanding of contributory risk factors including diet was high; however awareness of exercise as a preventative measure was poor with only $7 \%$ of participants reporting their children to be involved in regular exercise. Despite this finding, 50\% felt that their children gained enough exercise. Knowledge of locally available interventions including healthy eating schemes and extra-curricular activities was highly variable with first-generation migrants displaying a complete lack of awareness.

Conclusions This study highlights a lack of awareness of preventative measures in addition to poor knowledge of available interventions. This suggests a need for promoting culturally sensitive obesity education and intervention planning in partnership with families of South Asian origin, especially amongst firstgeneration migrants. 